\title{
SLC5A5 Gene
}

National Cancer Institute

\section{Source}

National Cancer Institute. SLC5A5 Gene. NCI Thesaurus. Code C20904.

This gene is involved in iodide transport. 